A Pharmacist Intervention In Very Old inpatients

Combined Use Of The Rationalization Of Home Medication By An Adjusted STOPP (RASP) List And A

Pharmacist-Led Medication Review In Very Old Inpatients: Impact On Quality Of Prescribing And

\title{
Clinical Outcome
}

Lorenz Van der Linden ${ }^{1,2}$, Liesbeth Decoutere ${ }^{3}$, Karolien Walgraeve ${ }^{1}$, Koen Milisen ${ }^{4}$, Johan Flamaing ${ }^{5,6}$, Isabel Spriet $^{1,2}$, Jos Tournoy ${ }^{5,6}$

1 Pharmacy Department, University Hospitals Leuven, Leuven, Belgium, ${ }^{2}$ Department of Pharmaceutical and Pharmacological Sciences, KU Leuven, Leuven, Belgium, ${ }^{3}$ Pharmacy Department, Jessa Hospital, Hasselt, Belgium, ${ }^{4}$ Department of Public Health and Primary Care, Health Services and Nursing Research, KU Leuven, Leuven, Belgium, ${ }^{5}$ Department of Geriatric Medicine, University Hospitals Leuven, Leuven, Belgium; ${ }^{6}$ Department of Clinical and Experimental Medicine, KU Leuven, Leuven, Belgium

Corresponding Author: Lorenz Van der Linden; e-mail: lorenz.vanderlinden@uzleuven.be ; address: University Hospitals Leuven, Pharmacy Department, Herestraat 49, 3000 Leuven, Belgium; phone: +3216343080; fax: +3216343085 


\section{ABSTRACT}

BACKGROUND: Polypharmacy and potentially inappropriate drugs have been associated with negative outcomes in older adults which might be reduced by pharmacist interventions.

OBJECTIVES: Our objective was to evaluate the effect of a pharmacist intervention, consisting of the application of the RASP list and a pharmacist-led-medication review on polypharmacy, the quality of prescribing and clinical outcome in geriatric inpatients.

METHODS: A monocentric, prospective controlled trial was undertaken at the geriatric wards of a large university hospital. Pharmacists applied The Rationalization Of Drugs By An Adjusted STOPP (RASP) list to the drugs reconciled upon admission and additionally performed an expert-based medication review, upon which recommendations were provided to the treating physicians. The primary outcome was the composite endpoint of drug discontinuation and dose reduction of drugs taken on admission. Secondary outcomes included RASPidentified potentially inappropriate medications (PIMs), the number of emergency department (ED) visits and quality-of-life (QoL) registered up to three months after discharge.

RESULTS: Patients ( $\mathrm{n}=172)$ took on average 10 drugs on admission and were 84.5 years (SD: 4.8) old. More drugs were discontinued or reduced in dose in the intervention group (control vs. intervention, median (IQR): 3 (2-5) vs. 5 (3-7), $\mathrm{P}<0.001)$. More PIMs were discontinued in the intervention group, leading to less PIM at discharge (control vs. intervention, median (IQR): 2 (1-3) vs. $0.5(0-1), \mathrm{P}<0.001)$. No signal of harm was seen; a significant improvement of QoL and less ED visits without hospitalization were observed.

CONCLUSIONS: The combined intervention safely reduced drug use in very old inpatients and outperformed usual geriatric care. An increased QoL was seen as well as a trend towards less ED visits. ClinicalTrials.gov Identifier: NCT01513265

\section{Key Points}

1. Polypharmacy and the use of potentially inappropriate drugs are both very common in patients admitted to acute geriatric wards.

2. Implementation of a pharmacist intervention in very old inpatients, which consisted of a medication review based on but not limited to the RASP list, was associated with a larger reduction of the preadmission drugs as compared to usual clinical care. This decrease in the number of drugs was off-set by recommendations to initiate certain drug therapies (e.g. calcium and vitamin D) during hospital stay. 
3. The intervention resulted in a significant decrease of the number of potentially inappropriate drugs at discharge.

4. No signal of harm was found during the study. An improvement of the quality of life and a signal of reduced emergency department visits was seen in favor of the intervention group.

\section{ACKNOWLEDGMENTS}

We wish to thank Siska Desplenter and Elien Brants for assisting in the follow-up of patients during hospital stay and also in performing follow-up telephone interviews.

\section{AUTHOR CONTRIBUTIONS}

Lorenz Van der Linden and Liesbeth Decoutere participated in study concept and design, acquisition of subjects and data, analysis and interpretation of data, and preparation of manuscript. Karolien Walgraeve participated in acquisition of subjects and data and in the preparation of the manuscript. Jos Tournoy, Isabel Spriet and Johan Flamaing participated in study concept and design, analysis of data and preparation of the manuscript. Koen Milisen participated in interpretation of data and preparation of the manuscript.

\section{COMPLIANCE WITH ETHICAL STANDARDS}

Funding

No sources of funding were used to assist in the conduct of this study or the preparation of this article.

\section{Conflicts of Interest}

Lorenz Van der Linden, Liesbeth Decoutere, Karolien Walgraeve, Koen Milisen, Johan Flamaing, Isabel Spriet and Jos Tournoy declare that they have no conflicts of interest relevant to the content of this article.

\section{Consent}

This trial was approved by the Ethical Committee of the University Hospital Leuven, Leuven, Belgium. Informed consent was obtained before enrollment into the study. 
A Pharmacist Intervention In Very Old inpatients

\section{INTRODUCTION}

Drug therapy is the mainstay of treatment for many conditions in very old adults [1-3]. At our institute very old people are defined as patients who are 75 years or older. The evidence supporting drug therapies in the very old is scant as they often were excluded from clinical trials, even for highly prevalent conditions [4]. Appropriate drug use in this age group has therefore been based substantially on expert opinion and extrapolations from trials performed in younger adults [5]. A high prevalence of comorbidities further compromise the applicability of the limited evidence. Furthermore, regarding the use of preventive drug therapies (e.g. statins) in older adults, the time-to-effect has not been sufficiently elucidated [6]. Also, therapy aims might change, as quality of life could be perceived as becoming more important than prolonging life at a certain point in life $[7,8]$.

While healthcare-providers intend to attain specific goals by prescribing drug therapies appropriately, polymorbidity and the complexity of the prescribing process in this population can lead to polypharmacy and inappropriate prescribing $[9,10]$. Polypharmacy and inappropriate prescribing have been associated with adverse drug events (ADE), increased health care expenditures and hospital admissions [3, 11-15].

Screening tools have been developed to assess inappropriate prescribing in older adults [16-18]. They encompass the overuse, misuse and/or underuse of drugs and these instruments differ in content, extensiveness and in the way they should be used [19]. Implicitly formulated instruments provide specific questions or a general algorithm, thereby aiding the experienced clinician in determining whether a specific drug should be considered inappropriate. Explicit criteria document potentially inappropriate drugs as such, whether or not taking into account certain circumstances (e.g. renal function) $[17,19,20]$. The implementation of an explicit screening tool to improve prescribing has already been shown to reduce the number of medications in older persons [21]. The causal relation between reducing potentially inappropriate medications (PIM) and improving clinical outcomes has however not yet been robustly demonstrated [22-25]. Recently, Verdoorn et al proposed that combining an explicit and implicit approach might further strengthen drug discontinuation efforts in older persons [26]. Adding pharmacists to the multidisciplinary team might also improve the overall quality of prescribing and potentially reduce negative outcomes associated with inappropriate drug use in older persons [27]. Pharmacist interventions can be a part of an multidisciplinary and multifaceted approach, in which several processes should be addressed, such as the systematic application of aforementioned screening tools, the support of computerized decision support systems, a standardized drug reconciliation performed on a regular basis and a multidisciplinary comprehensive geriatric assessment [28]. 
In a previous investigation our group developed and validated the RASP list (Rationalization of home medication by an Adjusted STOPP list in older Patients), an iteration of the STOPP criteria (Screening Tool of Older Persons' potentially inappropriate Prescriptions) [16, 29]. The RASP list consists of 76 items divided over 12 groups, of which about one third was directly based on the STOPP criteria. It was developed to improve the capture rate of PIMs compared to available tools in geriatric patients, being at least 75 years of age.

In this study, we aimed to assess the effect of a pharmacist intervention (based on but not limited to the RASP list) on polypharmacy, inappropriate prescribing and clinical outcomes in older inpatients admitted to acute geriatric wards.

\section{METHODS}

\subsection{Setting and study design}

The study was performed on three acute geriatric wards of a 2000-bed university hospital in Flanders, Belgium. The study was performed in the geriatric inpatient population, i.e. very old patients, as opposed the ambulatory or clinic setting due to the access to medical files by hospital pharmacists and improved monitoring (i.e. during hospital stay) to determine any potential harm associated with the pharmacist intervention. Limiting the trial to the hospital setting also provided more opportunities to include more frail patients, i.e. there was more time to provide information in order to obtain an informed consent.

Allocation to the intervention versus (vs.) control arm was based on consecutive admissions to one control and two intervention wards. No active randomization was performed. There was no selection of patient profiles being admitted to a specific ward.

The study was approved by the local Ethics Committee and patients were only considered for inclusion if written informed consent was given by the patients, or their relatives in case of inability to provide the consent themselves (ClinicalTrials.gov Identifier NCT01513265).

\subsection{Blinding}

Geriatricians in the control group were not informed about the study design. If potentially life-threatening drug errors were observed, this was communicated to the treating physician as part of medication reconciliation services provided in both groups. 
A Pharmacist Intervention In Very Old inpatients

\subsection{Patients}

Dutch-speaking patients admitted from home or from a nursing home were eligible for inclusion. Patients were excluded if admitted for end-of-life care, if they did not take any drugs or if they were not discharged back to their home or a nursing home. If end-of-life-care was instituted during hospital stay, enrolled patients were excluded from analysis to prevent bias induced by the systematic drug discontinuation (regardless the appropriateness) in these patients; the RASP list was not be used in patients in whom end-of-life care was initiated.

\section{Baseline data collection}

Preadmission medication was reconciled by a clinical pharmacist using a standardized medication reconciliation sheet [30]. A clinical pharmacist then applied the RASP list on all admission and discharge drug therapies; PIMs identified by the RASP were documented (i.e. RASP PIMs).

The following patient characteristics were collected: age, sex, weight (kg), the 6-item Katz index of activities of daily living, the Charlson comorbidity score (CCS) and the quality of life (QoL), by using the European Quality of Life-5 Dimensions Questionnaire (EQ-5D-3L) questionnaire [31-33].

\subsection{Intervention}

The intervention consisted of trained clinical pharmacists (EB, SD, KW, LD, LVDL), performing medication reconciliation with a subsequent two-stage medication review. The reconciled drug information was registered in the electronic patient file. In a first step of the medication review, the RASP list was applied [29]. All pharmacists were trained in the use of RASP list after having received the necessary introductory courses by the senior pharmacists (LVDL, LD). A second step comprised an additional comprehensive medication review by the clinical pharmacist, covering mistreatment (e.g. use of antipsychotic drugs in the treatment of agitation in patients suffering from dementia), overtreatment (e.g. prolonged use of proton-pump inhibitors) as well as potential undertreatment (e.g. no use of anticoagulation in atrial fibrillation). Training of the clinical pharmacists was overseen by senior pharmacists (LVDL, LD) and geriatricians (JT, JF). Senior pharmacists (LVDL, LD) attended ward rounds systematically to ensure correct study conduct. 
In the intervention group, recommendations were actively reported on a daily basis to the treating physician. It was left to the discretion of the treating physician, whether to follow the pharmaceutical recommendations. Accepted recommendations were included in the discharge letter to the general practitioner.

\subsection{Outcome measures}

The primary outcome was the number of admission drugs discontinued or reduced in dose on admission.

Following secondary outcomes were registered during hospital stay: the number of discontinued and started drugs, drugs reduced in dose, the prevalence of delirium assessed by bedside nurses using the Delirium Observation Scale and confirmed by the treating geriatrician, the number of falls registered by the nurses using the hospitals' incident report system, in-hospital mortality and the number of RASP PIMs [34]. The ratio of discontinued and/or reduced drugs during hospital stay to the number of drugs taken on admission was reported as well. Likewise, the proportion of discontinued or reduced drugs which was identified by the RASP list was calculated.

Following secondary outcomes were registered after discharge. Patients and their primary-care physicians were contacted by phone, one and three months after hospital discharge to verify drug therapy. The numbers of allcause readmissions to the hospital and emergency department (ED) visits, i.e. with and without subsequent hospitalizations, were documented. All-cause ED visits were determined after considering their importance in the clinical trajectory of the patient and their expected amenability to our type of intervention, as previously shown by Gillespie et al [35].

The mortality rate within three months after discharge was documented as well, as were the number of falls and QoL. If patients experienced a fall during the three-month follow-up, fall-related injuries were determined and were defined as minor (i.e. fall with no or only limited medical consequence (e.g. small hematoma, scratch)) or major (e.g. fractures, loss of consciousness, severe hematoma).

\subsection{Sample size}

A sample size of 34 patients per group was needed to detect a difference with $80 \%$ power in the primary outcome (i.e. the number of discontinued or reduced chronic drugs) using the Mann-Whitney U (MWU) test (two-tailed, alpha: 0.05, Cohen’s d: 0.7). Moreover, 81 patients were needed in each group to detect a difference in the ratio of drugs at discharge to the number on admission using the MWU test (two-tailed, alpha: 0.05, 
Cohen's d: 0.45 ). Bearing in mind a potential $20 \%$ loss of inclusions due to attrition, 203 patients needed to be enrolled in total.

\subsection{Comparison of the RASP list to other tools}

In a post-hoc analysis, the RASP list was compared to other screening tools. The 2012 Beers criteria, and the 2008 STOPP list were applied to the drug charts reconciled on admission from all patients [16, 36]. The number of PIM identified by the STOPP or Beers criteria was determined, as well as the number of applicable criteria of both screening tools.

\subsection{Statistical analysis}

Normality was determined by performing the Kolmogorov-Smirnov test and by visual examination of the histograms. The Fisher Exact was used for categorical variables, the Student $t$-test for normally distributed continuous variables, and the MWU test for non-normally distributed continuous variables.

Linear mixed modeling (LMM) was used to ascertain the impact of the intervention, the time course after discharge and a potential interaction between the former two. The analysis was modeled for the following dependent continuous variables: number of drugs with and without calcium and vitamin D therapy. The correction for calcium and vitamin D therapy was not predefined and was performed to account for the fact that calcium and vitamin D had been initiated significantly more often in the intervention group as it was frequently recommended by the pharmacists as part of their medication review.

Time (at discharge and one and three months after discharge) and study arm allocation (control vs. intervention) were used as fixed factors in the model. Random effects pertained to the individual subjects; a random intercept was defined at the patient level. If no significant interaction between time and group was seen, the main effects results were reported.

The same LMM approach was applied to the QoL data (EQ-5D-3L), which were collected on admission (at baseline) and at one and three months after discharge. The questionnaire results were converted to a numerical EQ-5D-3L index (0 to 1; 0 indicating dead and 1 a healthy state) per measurement per patient, which was then used as the dependent continuous variable in the linear mixed model [37]. 
Statistical significance was established as $\mathrm{P}<0.05$, with all tests being two-tailed. No adjustment for multiple comparisons were made. Statistical analyses were executed with IBM SPSS Statistics for Windows Version 20.0 (Armonk, NY: IBM Corp). Sample size estimations were calculated using G*Power 3.1 [38].

\section{RESULTS}

\subsection{Study population}

During the 11 month study course, 438 patients were admitted, of which 224 refused participation or did not meet enrollment criteria. Figure 1 depicts the flow of the study participants [39]. The remaining consenting 214 subjects were successfully assigned to the intervention $(n=117)$ or control group $(n=97)$. At discharge, data of 172 patients, who fulfilled the inclusion and exclusion criteria, with an average age of 84.5 (SD: 4.8) years remained available for analysis (see Figure 1). Table 1 shows the baseline characteristics of both groups. No significant differences were found between the two study arms.

\subsection{Primary outcome measures}

On admission, an average of two more drugs were discontinued or reduced in dose in the intervention group $(\mathrm{P}=0.001)$ (Table 2). Taking into account the number of drugs reconciled upon admission, $18 \%$ more drugs were discontinued or reduced in dose in the intervention group (control vs. intervention, median (IQR): 0.32 (0.21$0.49)$ vs. $0.50(0.42-0.63), \mathrm{P}<0.001)$

\subsection{Secondary outcome measures}

At discharge, no significant differences were observed concerning the ratio of the number of drugs to the number of drugs on admission (Table 2). After the initial discontinuation, more drugs were initiated in the intervention group during hospital stay (control vs. intervention, median (IQR): 2 (1-3) vs. 3 (2-4), P<0.001). Most prominently, calcium and vitamin D therapy was initiated significantly more in the intervention group (18/81 in the control vs. 56/91 in the intervention group, $\mathrm{P}<0.001$ ). Adjustment for all or de novo calcium and vitamin $\mathrm{D}$ therapy rendered the result statistically significant, resulting in $16 \%$ fewer drugs at discharge in the intervention group $(\mathrm{P}=0.017)$ (Table 2).

More RASP PIMs were stopped and not (re)initiated during hospital stay in the intervention vs. control group, leading to a lower number of RASP PIMs in the intervention group (control vs. intervention, median (IQR): 2 (13) vs. $0.5(0-1), \mathrm{P}<0.001)$ at discharge. Three specific RASP PIMs were significantly discontinued more often in 
the intervention group: use of nitrates/NO-donors without a compelling indication, long-term use of antidepressants, long-term use of proton-pump inhibitors.

One month after discharge full data were collected for 75 and 85 study subjects in the control and intervention group respectively and at three months after discharge for 68 and 82 patients. During hospital stay and after one and three months, no significant differences were observed for the number of falls or mortality rate. During the follow-up period, no significant difference was seen in the number of readmissions or emergency admissions. A statistically significant difference in the number of ED visits without subsequent hospitalization was seen in favor of the intervention group $(\mathrm{P}=0.021)$ (Table 3).

\subsection{1. $\quad$ Drug use after discharge}

The estimated average number of drugs after discharge was 8.86 (standard error (SE): 0.21) and 9.37 (SE: 0.23) in the intervention and control group respectively. The intervention did not decrease overall drug use after discharge compared to usual care (mean difference: 0.52 (SE: 0.31, $\mathrm{P}=0.098$ ). The estimated means used in the main effects analysis have been depicted in Figure 2. No significant interaction between time and group was found.

A correction for calcium and vitamin D therapy resulted in a significant decrease of the number of drugs after discharge (control vs. intervention, estimated mean (SE): 8.28 (0.22) vs. $7.20(0.21), \mathrm{P}<0.001)$.

\subsubsection{Quality of Life}

At none of the three time points at which the EQ-5D-3L was determined, a significant difference of the components was found between both groups. Main effects analysis showed that subjects in the intervention group experienced an increased QoL with an average EQ-5D index of 0.358 (SE: 0.016) vs. 0.294 (SE: 0.018) in the control group, denoting a difference of 0.064 points (SE: $0.024, \mathrm{P}=0.008$ ) in favor of the intervention group. No significant interaction between time and group was found.

\subsubsection{Post-hoc comparison to STOPP and Beers criteria}

Data of all 172 admitted patients were analyzed. The main involved drug classes were similar for the three tested instruments: benzodiazepines, antipsychotics and antidepressants, accounting for $43.6 \%$ of the Beers criteria ( $n=408), 26.0 \%$ of the STOPP items $(n=288)$, and $33.8 \%$ of the RASP list $(n=640)$.
Commented [LVDL1]: Categorische analyse: fisher exact van stoppen/statusquo/opgestart. 
On average, the RASP list identified 1.8 (95\% confidence interval (CI): 1.5, 2.2, P<0.001) and 1.6 (95\% CI 1.4, 1.9, $\mathrm{P}<0.001$ ) more PIM per patient on admission when compared to the STOPP and Beers criteria respectively. Likewise, more RASP items per patient could be applied than Beers criteria (mean difference: 2.9; 95\% CI: 2.5, 3.4, $\mathrm{P}<0.001$ ) or STOPP criteria (mean difference: 3.7; 95\% CI: 3.2, 4.3, $\mathrm{P}<0.001$ ).

\section{DISCUSSION}

A new combined intervention resulted in the safe discontinuation of pre-admission drugs in very old inpatients. On admission, an average of two more drugs was discontinued or reduced in dose, i.e. an extra $18 \%$ of the reconciled drugs, compared to usual care. The decrease in the primary composite endpoint was almost exclusively driven by a significant difference in the proportion of drug discontinuation in both groups.

We also observed a slight yet statistically significant increase in the number of drugs initiated in the intervention group during hospital stay, which was almost exclusively driven by the increased initiation of calcium and vitamin D. Hence, drug discontinuation upon admission resulted in a decrease of $16 \%$ in the total number of drugs at discharge, but only after adjusting for calcium and vitamin D therapy. Furthermore, LMM analysis showed that the decrease in the number of drugs persisted after discharge, yet again, only after correcting for calcium and vitamin D therapy. A small trend towards an increase was seen in the number of drugs in both groups after discharge, because of re-initiation by the general practitioner.

RASP PIM were discontinued more often and not (re)initiated in the intervention group, leading to fewer PIM at discharge, underlying the usefulness of the RASP list as a crucial component of the intervention. Given the extent of the RASP list (i.e. it contains 76 items), the study was not powered to detect differences in specific

RASP PIMs. Nonetheless, three classes could be identified that were discontinued significantly more often without having being restarted in the intervention group: antidepressants, proton-pump inhibitors and nitrates/NO-donors. In a post-hoc analysis more PIM were also identified by the RASP list as compared to the Beers 2012 and the original STOPP START criteria [16, 36]. The analysis was not repeated with the new renditions of these criteria as the improved sensitivity in our study was mostly explained by the more inclusive wording of the RASP list and not by the drug classes involved. The tested instruments had similar top three PIMs, including inappropriate benzodiazepine, antidepressant and antipsychotic drug use. All tools were however inherently limited in their ability to detect inappropriate prescribing in older adults; only about half of the discontinued drugs was detected by the RASP list (Table 2) [40]. The remainder of the discontinued drugs was diverse (i.e. low prevalence per situation in which a specific drug was discontinued); a large portion was due 
to general overtreatment, e.g. very strict glycated haemoglobin (HbA1C) values under sulfonylurea therapy. Nonetheless, exhaustive and hence time-consuming tools might yet prove to be useful in daily practice, if offered as part of clinical decision support software (e.g. the STRIP assistant) [41]. Our data support the notion however that a thorough medication review in a heterogeneous acutely ill older population requires the combination with an expert-based (or implicit) approach; experts have been shown to systematically outperform explicit tools [40, 42].

The intervention was associated with a significant increase of the EQ-5D-3L index with 0.064 points $(\mathrm{P}=0.008)$, in contrast to previous findings [43, 44]. The preference-based EQ-5D-3L questionnaire allowed for a valid determination of the QoL in this aged group [45, 46]. Although our results are reassuring and statistically significant, the difference remained small and in the range of the average minimally important difference for the EQ index, being 0.074 [47]. Furthermore, the difference could have been influenced by many unmeasured covariates, for which the study was neither designed nor controlled to detect.

No statistically significant differences were seen in any of the other secondary clinical outcome measures. A trend was observed however in the number of patients visiting the emergency department at least once, in favor of the intervention group (39.2\% vs. 28.7\%; $\mathrm{P}=0.189$ ). Particularly, a significant difference was found in the number of ED visits without hospital admission $(\mathrm{P}=0.02)$, which might be explained by a lower incidence of adverse drug events or an improved health literacy. Our results are in line with data from a recent meta-analysis performed by Christensen et al, which suggested that a medication review, performed during hospital stay, could decrease incidental emergency department contacts [23]. Confirmatory trials are surely needed and will most likely target all-cause ED visits, avoidable readmissions to the hospital or drug-related readmissions.

Although hospital stay offers a period of increased patient monitoring, the impact of the review on clinical outcomes might have been diminished due to the short-lived nature of the intervention [48, 49]. An intervention, promoting a closer involvement of primary care professionals and patients, might result in a larger persistence of the effect on drug use after discharge. Along that line and in an outpatient setting, a systematic deprescribing intervention was investigated in a recent, uncontrolled study of Garfinkel et al in which they showed a discontinuation rate of 58\%, which persisted during the one-year follow-up [50]. We hypothesize that a longer and more extensive intervention would further reduce the iatrogenic drug burden within this group; extending the time frame of follow-up by the same team (e.g. up to three months), a more intensive involvement of patients 
and family and a systematic collaboration with primary care providers might provide more leverage to institute an effective (de)prescribing plan [50,51].

Broad inclusion criteria, the unselected 'real-life' study sample, the longitudinal follow-up, a low loss of patients due to attrition and the prospective controlled design of this study add to the validity of our study results. As the control group was managed by an experienced geriatrician team (i.e. 32\% of drugs on admission was discontinued), the effect size of the intervention might prove to be much larger in a very old inpatients in nongeriatric setting. This is potentially relevant, given the high bed occupancy by older adults in hospitals, e.g. in our hospital, patients 80 years and older occupy on average $10 \%$ of all hospital beds.

Our study however has certain limitations. Because of the teaching hospital setting with a substantial turnover of physicians-in-training and the nature of our intervention, no parallel randomized controlled trial design was undertaken. No a priori selection occurred however when admitting patients to the wards; patients were enrolled consecutively and no relevant differences were seen at baseline. Furthermore, follow-up of patients was limited to three months after discharge. However, this time frame offers primary care physicians sufficient time to read the discharge letters, detect changes in drug use and adjust drug therapies at their own discretion. We also did not prospectively determine the specific cause of readmission or ED visit, in terms of them being related to drugs or not; despite this limitation, a benefit towards less all-cause ED visits was seen in favor of the intervention group.

Further research is urgently needed to confirm and refine our findings, especially concerning the effect of the intervention on clinically relevant outcomes, such as visits to the emergency department. This investigation has directly led to several follow-up research projects that all took place in our hospital, such as the prospective cohort study in which the added value of a hospital pharmacist in the care of very old heart failure inpatients was determined. Afterwards a quasi-experimental study was undertaken in collaboration with the geriatric consultation team to establish the impact of using the RASP list in very old patients, not admitted to acute geriatric wards. Currently, our group is involved in promoting transmural care and a large project in primary care to reduce unplanned admissions in high risk ambulatory old persons.

\section{CONCLUSIONS}

A combined intervention safely reduced pre-admission drug use in very old inpatients and outperformed usual geriatric care and alternative explicit criteria. More RASP PIMs were discontinued in the intervention group, leading to less PIMs at discharge. Patients were discharged with less drugs in the intervention group, after 
correcting for calcium and vitamin D therapy. An increase in the quality of life was seen, as well as a trend towards less emergency department visits.

\section{REFERENCES}

1. Hovstadius B, Hovstadius K, Astrand B, Petersson G. Increasing polypharmacy - an individual-based study of the Swedish population 2005-2008. BMC Clin Pharmacol. England; 2010. p. 16.

2. Rochon PA, Gurwitz JH. Optimising drug treatment for elderly people: the prescribing cascade. BMJ. 1997 Oct 25;315(7115):1096-9.

3. Payne RA, Avery AJ. Polypharmacy: one of the greatest prescribing challenges in general practice. Br J Gen Pract. 2011 Feb;61(583):83-4.

4. Cherubini A, Oristrell J, Pla X, Ruggiero C, Ferretti R, Diestre G, et al. The persistent exclusion of older patients from ongoing clinical trials regarding heart failure. Arch Intern Med. United States; 2011. p. 5506.

5. Wehling M. Guideline-driven polypharmacy in elderly, multimorbid patients is basically flawed: there are almost no guidelines for these patients. J Am Geriatr Soc. 2011 Feb;59(2):376-7.

6. Holmes HM, Min LC, Yee M, Varadhan R, Basran J, Dale W, et al. Rationalizing prescribing for older patients with multimorbidity: considering time to benefit. Drugs Aging. 2013 Sep;30(9):655-66.

7. Brunner-La Rocca HP, Rickenbacher P, Muzzarelli S, Schindler R, Maeder MT, Jeker U, et al. End-oflife preferences of elderly patients with chronic heart failure. Eur Heart J. England; 2012. p. 752-9.

8. Gheorghe C, Vazquez R, Casanegra AI, Argento V, Dadu R, Feng Y, et al. Elders' environs and their end-of-life preferences. J Am Med Dir Assoc. United States: 2011 American Medical Directors Association. Published by Elsevier Inc; 2011. p. 22-8.

9. Gallagher P, Barry P, O'Mahony D. Inappropriate prescribing in the elderly. J Clin Pharm Ther. 2007 Apr;32(2):113-21.

10. Boyd CM, Darer J, Boult C, Fried LP, Boult L, Wu AW. Clinical practice guidelines and quality of care for older patients with multiple comorbid diseases: implications for pay for performance. JAMA. United States; 2005. p. 716-24.

11. Cahir C, Bennett K, Teljeur C, Fahey T. Potentially inappropriate prescribing and adverse health outcomes in community dwelling older patients. Br J Clin Pharmacol. 2014 Jan;77(1):201-10.

12. Laroche ML, Charmes JP, Nouaille Y, Picard N, Merle L. Is inappropriate medication use a major cause of adverse drug reactions in the elderly? Br J Clin Pharmacol. England; 2007. p. 177-86. 
13. Mannesse CK, Derkx FH, de Ridder MA, Man in 't Veld AJ, van der Cammen TJ. Contribution of adverse drug reactions to hospital admission of older patients. Age Ageing. 2000 Jan;29(1):35-9.

14. Schoenenberger AW, Stuck AE. Inappropriate drug use among older persons: is it time for action? Age Ageing. England; 2014. p. 737-9.

15. Maher RL, Hanlon J, Hajjar ER. Clinical consequences of polypharmacy in elderly. Expert Opin Drug Saf. 2014 Jan;13(1):57-65.

16. Gallagher P, Ryan C, Byrne S, Kennedy J, O'Mahony D. STOPP (Screening Tool of Older Person's Prescriptions) and START (Screening Tool to Alert doctors to Right Treatment). Consensus validation. Int J Clin Pharmacol Ther. 2008 Feb;46(2):72-83.

17. Samsa GP, Hanlon JT, Schmader KE, Weinberger M, Clipp EC, Uttech KM, et al. A summated score for the medication appropriateness index: development and assessment of clinimetric properties including content validity. J Clin Epidemiol. 1994 Aug;47(8):891-6.

18. Pretorius RW, Gataric G, Swedlund SK, Miller JR. Reducing the risk of adverse drug events in older adults. Am Fam Physician. 2013 Mar 1;87(5):331-6.

19. Spinewine A, Schmader KE, Barber N, Hughes C, Lapane KL, Swine C, et al. Appropriate prescribing in elderly people: how well can it be measured and optimised? Lancet. 2007 Jul 14;370(9582):173-84.

20. Fick DM, Cooper JW, Wade WE, Waller JL, Maclean JR, Beers MH. Updating the Beers criteria for potentially inappropriate medication use in older adults: results of a US consensus panel of experts. Arch Intern Med. 2003 Dec 8-22;163(22):2716-24.

21. Frankenthal D, Lerman Y, Kalendaryev E. Intervention with the screening tool of older persons potentially inappropriate prescriptions/screening tool to alert doctors to right treatment criteria in elderly residents of a chronic geriatric facility: a randomized clinical trial. J Am Geriatr Soc. 2014 Sep;62(9):1658-65.

22. Nobili A, Licata G, Salerno F, Pasina L, Tettamanti M, Franchi C, et al. Polypharmacy, length of hospital stay, and in-hospital mortality among elderly patients in internal medicine wards. The REPOSI study. Eur J Clin Pharmacol. 2011 May;67(5):507-19.

23. Christensen M, Lundh A. Medication review in hospitalised patients to reduce morbidity and mortality. Cochrane Database Syst Rev. 2013;2:Cd008986.

24. Tjia J, Velten SJ, Parsons C, Valluri S, Briesacher BA. Studies to reduce unnecessary medication use in frail older adults: a systematic review. Drugs Aging. 2013 May;30(5):285-307. 
25. Gillespie U, Alassaad A, Hammarlund-Udenaes M, Morlin C, Henrohn D, Bertilsson M, et al. Effects of pharmacists' interventions on appropriateness of prescribing and evaluation of the instruments' (MAI, STOPP and STARTs') ability to predict hospitalization--analyses from a randomized controlled trial. PLoS One. 2013;8(5):e62401.

26. Verdoorn S, Kwint HF, Faber A, Gussekloo J, Bouvy ML. Majority of drug-related problems identified during medication review are not associated with STOPP/START criteria. Eur J Clin Pharmacol. 2015 Oct;71(10):1255-62.

27. Renaudin P, Boyer L, Esteve MA, Bertault-Peres P, Auquier P, Honore S. Do pharmacist-led medication reviews in hospitals help reduce hospital readmissions? A systematic review and meta-analysis. Br J Clin Pharmacol. 2016 Aug 11.

28. Petrovic M, Somers A, Onder G. Optimization of Geriatric Pharmacotherapy: Role of Multifaceted Cooperation in the Hospital Setting. Drugs Aging. 2016 Mar;33(3):179-88.

29. Van Der Linden L, Decoutere L, Flamaing J, Spriet I, Willems L, Milisen K, et al. Development and validation of the RASP list (Rationalization of Home Medication by an Adjusted STOPP list in Older Patients): A novel tool in the management of geriatric polypharmacy. European Geriatric Medicine. 2014;5(3):175-80.

30. De Winter S, Vanbrabant P, Spriet I, Desruelles D, Indevuyst C, Knockaert D, et al. A simple tool to improve medication reconciliation at the emergency department. Eur J Intern Med. 2011 Aug;22(4):382-5.

31. Katz S, Akpom CA. 12. Index of ADL. Med Care. 1976 May;14(5 Suppl):116-8.

32. Charlson ME, Pompei P, Ales KL, MacKenzie CR. A new method of classifying prognostic comorbidity in longitudinal studies: development and validation. J Chronic Dis. 1987;40(5):373-83.

33. EuroQol--a new facility for the measurement of health-related quality of life. Health Policy. 1990 Dec;16(3):199-208.

34. Schuurmans MJ, Shortridge-Baggett LM, Duursma SA. The Delirium Observation Screening Scale: a screening instrument for delirium. Res Theory Nurs Pract. 2003 Spring;17(1):31-50.

35. Gillespie U, Alassaad A, Henrohn D, Garmo H, Hammarlund-Udenaes M, Toss H, et al. A comprehensive pharmacist intervention to reduce morbidity in patients 80 years or older: a randomized controlled trial. Arch Intern Med. United States; 2009. p. 894-900.

36. American Geriatrics Society updated Beers Criteria for potentially inappropriate medication use in older adults. J Am Geriatr Soc. 2012 Apr;60(4):616-31. 
37. Cleemput I. A social preference valuations set for EQ-5D health states in Flanders, Belgium. Eur J Health Econ. 2010 Apr;11(2):205-13

38. Faul F, Erdfelder E, Lang AG, Buchner A. G*Power 3: a flexible statistical power analysis program for the social, behavioral, and biomedical sciences. Behav Res Methods. 2007 May;39(2):175-91

39. Altman DG, Schulz KF, Moher D, Egger M, Davidoff F, Elbourne D, et al. The revised CONSORT statement for reporting randomized trials: explanation and elaboration. Ann Intern Med. United States; 2001. p. 663-94.

40. Steinman MA, Rosenthal GE, Landefeld CS, Bertenthal D, Kaboli PJ. Agreement between drugs-toavoid criteria and expert assessments of problematic prescribing. Arch Intern Med. United States; 2009. p. 1326-32.

41. Meulendijk MC, Spruit MR, Drenth-van Maanen AC, Numans ME, Brinkkemper S, Jansen PA, et al. Computerized Decision Support Improves Medication Review Effectiveness: An Experiment Evaluating the STRIP Assistant's Usability. Drugs Aging. 2015 Jun;32(6):495-503.

42. Marriott J, Stehlik P. A critical analysis of the methods used to develop explicit clinical criteria for use in older people. Age Ageing. 2012 Jul;41(4):441-50.

43. Krska J, Cromarty JA, Arris F, Jamieson D, Hansford D, Duffus PR, et al. Pharmacist-led medication review in patients over 65: a randomized, controlled trial in primary care. Age Ageing. 2001 May;30(3):205-11.

44. Hanlon JT, Weinberger M, Samsa GP, Schmader KE, Uttech KM, Lewis IK, et al. A randomized, controlled trial of a clinical pharmacist intervention to improve inappropriate prescribing in elderly outpatients with polypharmacy. Am J Med. 1996 Apr;100(4):428-37.

45. Bulamu NB, Kaambwa B, Ratcliffe J. A systematic review of instruments for measuring outcomes in economic evaluation within aged care. Health Qual Life Outcomes. 2015;13:179.

46. Payakachat N, Ali MM, Tilford JM. Can The EQ-5D Detect Meaningful Change? A Systematic Review. Pharmacoeconomics. 2015 Nov;33(11):1137-54.

47. Walters SJ, Brazier JE. Comparison of the minimally important difference for two health state utility measures: EQ-5D and SF-6D. Qual Life Res. 2005 Aug;14(6):1523-32.

48. Gallagher PF, O'Connor MN, O'Mahony D. Prevention of potentially inappropriate prescribing for elderly patients: a randomized controlled trial using STOPP/START criteria. Clin Pharmacol Ther. United States; 2011. p. 845-54. 
A Pharmacist Intervention In Very Old inpatients

49. Borne R, Cumbler E, Glasheen JJ. Reducing Polypharmacy: Is Hospitalization the Right Time? Archives of Internal Medicine. 2011 2011/05/09;171(9):869-70.

50. Garfinkel D, Mangin D. Feasibility study of a systematic approach for discontinuation of multiple medications in older adults: addressing polypharmacy. Arch Intern Med. 2010 Oct 11;170(18):1648-54.

51. Scott IA, Anderson K, Freeman CR, Stowasser DA. First do no harm: a real need to deprescribe in older patients. Med J Aust. Australia; 2014. p. 390-2. 